\title{
Src-Dependent Tyrosine Phosphorylation at the Tips of Growth Cone Filopodia Promotes Extension
}

\author{
Estuardo Robles, Stephanie Woo, and Timothy M. Gomez \\ Department of Anatomy and Neuroscience Training Program, University of Wisconsin, Madison, Wisconsin 53706
}

\begin{abstract}
Extracellular cues guide axon outgrowth by activating intracellular signaling cascades that control the growth cone cytoskeleton. However, the spatial and temporal coordination of signaling intermediates remains essentially unknown. Live imaging of tyrosine phosphorylation in growth cones revealed dynamic phospho-tyrosine (PY) signals in filopodia that directly correlate with filopodial behavior. Local PY signals are generated at distal tips of filopodia during extension and are lost during retraction. Active Src family kinases localize to the tips of filopodia, and Src activity regulates both filopodial dynamics and local PY signaling. Positive guidance cues stimulate filopodial motility by locally increasing tyrosine phosphorylation in a cell division cycle 42 (Cdc42)-dependent manner. Locally reduced Src activity on one side of the growth cone generates an asymmetry in filopodial motility and PY signaling that promotes repulsive turning, suggesting that local changes in filopodial PY levels may underlie growth cone pathfinding decisions. p21-activated kinase (PAK), a Cdc42 effector whose activity is regulated by Src phosphorylation, also localizes to the tips of extending filopodia and controls filopodial motility. Coordinated activation of cytoskeletal effector proteins by GTPase binding and Src-mediated tyrosine phosphorylation may function to produce specific growth cone behaviors in response to guidance cues.
\end{abstract}

Key words: axon guidance; phospho-tyrosine; p21-activated kinase; Cdc42; actin; filopodia

\section{Introduction}

During axon pathfinding, growth cones extend filopodia that sample the environment for guidance cues. Studies performed in vitro suggest that asymmetric extension of filopodia underlies chemotropic growth cone turning in gradients of diffusible factors (Zheng et al., 1996). Consistent with an instructive role for filopodia during axon guidance, elimination of filopodia in vivo leads to pathfinding errors (Bentley and Toroian-Raymond, 1987; Chien et al., 1993). Although the function of individual filopodia as discrete signaling compartments has been demonstrated previously (Davenport et al., 1993; Gomez et al., 2001), the signals regulating filopodial formation and motility downstream of receptor activation by guidance cues are not well understood.

Filopodial extension is driven by polymerization of actin filaments at their tips (Mallavarapu and Mitchison, 1999), although the mechanisms regulating this process are unclear. Electron microscopic studies have revealed a filopodial tip complex located at the interface between growing ends of actin filaments and the plasma membrane (Lewis and Bridgman, 1992; Svitkina et al., 2003). Several components of this tip complex have been identified, including Ena/vasodilator-stimulated phosphoprotein actin-binding proteins (Lanier et al., 1999; Svitkina et al., 2003),

Received March 7, 2005; revised July 18, 2005; accepted July 18, 2005.

This work was supported by National Institutes of Health Grant NS41564 (T.M.G.) and a National Science Foundation predoctoral fellowship (E.R.). We thank Nick Spitzer, Bill Bement, Kate Kalil, and members of the Gomez laboratory for comments on this manuscript.

Correspondence should be addressed to Timothy M. Gomez, University of Wisconsin, 257 Bardeen Labs, 1300 University Avenue, Madison, WI 53706. E-mail: tmgomez@wisc.edu.

DOI:10.1523/JNEUROSCI.2680-05.2005

Copyright $\odot 2005$ Society for Neuroscience $\quad$ 0270-6474/05/257669-13\$15.00/0 the motor proteins myosin X and IIIa (Berg et al., 2002; Les Erickson et al., 2003), and $\beta 1$-integrins (Wu et al., 1996). In addition, Wu and Goldberg (1993) identified a robust enrichment of tyrosine-phosphorylated proteins at the tips of growth cone filopodia. Although initially thought to inhibit filopodial extension, these findings were inconsistent with pharmacological experiments indicating that tyrosine kinase activity promotes axon extension in vitro and in vivo (Gomez et al., 1996; Worley and Holt, 1996). Given that many guidance cues regulate axon growth and guidance by altering filopodial motility, it is important to understand the function of tyrosine phosphorylation at filopodial tips.

The Rho-family GTPase, cell division cycle 42 (Cdc42) is known to promote filopodial formation (Nobes and Hall, 1995). Cdc42 activity is also required for growth cone chemotropism toward BDNF (Yuan et al., 2003) and filopodial formation in response to netrin (Shekarabi and Kennedy, 2002). Recent studies have identified neural Wiskott-Aldrich syndrome protein (NWASP) and p21-activated kinase (PAK) as cytoskeletal regulators that are synergistically activated by $\mathrm{Cdc} 42$ and Src tyrosine kinases (Renkema et al., 2002; Torres and Rosen, 2003). The accumulation of tyrosine-phosphorylated proteins at the tips of filopodia and the prominent role of $\mathrm{Cdc} 42$ in filopodial formation together suggest that Src phosphorylation may locally regulate the activity of Cdc42 effectors involved in filopodial dynamics. However, it is uncertain whether either of these Cdc42 effectors function at the tips of growth cone filopodia.

Using a fluorescent protein indicator to visualize phosphotyrosine (PY) dynamics in living neurons, we found that tyrosine phosphorylation at the tips of growth cone filopodia promotes their formation and extension. Local PY signals are mediated by 
Src family kinases and are modulated by guidance cues in a Cdc42-dependent manner. Active Src colocalizes with Cdc42 at the tips of extending filopodia, suggesting that these signals may synergistically activate components of the filopodial tip complex. We further identify PAK, a target of both Src kinases and Cdc42, as a regulator of filopodial dynamics.

\section{Materials and Methods}

Embryo injection and cell culture. All expression constructs were subcloned into the pCS2 + vector for RNA synthesis (Dave Turner, University of Michigan, Ann Arbor, MI). Yellow fluorescent protein-Src homology-2 domain ( $\mathrm{dSH} 2$ ) construct was provided by Benjamin Geiger (Weizmann Institute, Rehovot, Israel). Human isoforms of mutant Cdc42 constructs [Q61L constitutively active and T17N dominantnegative (DN)] in pCS2 + were provided by Maureen Ruchhoeft. Human isoforms of wild-type and mutant PAK1 constructs were provided by Jonathan Chernoff (Fox Chase Cancer Center, Philadelphia, PA). Chick isoforms of kinase-dead (KD) (K297R) and constitutively active (CA) (Y527F) c-Src were provided by Patricia Keely (University of Wisconsin-Madison, Madison, WI) and Jonathan Cooper (Fred Hutchinson Cancer Research Center, Seattle, WA). One or two blastomeres at the eight-cell stage were injected with $0.1-1 \mathrm{ng}$ of capped mRNA transcribed in vitro (mMessage machine; Ambion, Austin, TX). For double-labeling experiments, embryos were coinjected with 50-100 ng of tetramethylrhodamine-dextran (TMR-D) (10,000 molecular weight; Molecular Probes, Eugene, OR) and mRNA. Neural tubes were dissected from 1-d-old embryos and cultured as described previously (Gomez et al., 2003). Neural tube explants were plated onto acid-washed coverslips coated with $50 \mu \mathrm{g} / \mathrm{ml}$ poly-D-lysine (Sigma, St. Louis, MO) or $25 \mu \mathrm{g} / \mathrm{ml}$ laminin (Sigma) and imaged or fixed $16-24 \mathrm{~h}$ after plating.

Confocal imaging. Fluorescent images of live and fixed cells were collected using a $60 \times$ (numerical aperture, 1.45) objective on an Olympus Optical (Tokyo, Japan) Fluoview 500 laser-scanning confocal system mounted on an AX-70 upright microscope. For double-label live imaging, sequential scanning was used to minimize fluorescence bleed through, resulting in an $\sim 1 \mathrm{~s}$ delay between green fluorescent protein (GFP) and TMR-D images. Rapid exchange of solutions was performed by sealing coverslips containing neuronal culture in an enclosed perfusion chamber with inlet and outlet ports. For immunocytochemistry, cultures were fixed in a $4 \%$ paraformaldehyde $/ 4 \%$ sucrose solution in $\mathrm{Ca} / \mathrm{Mg}$-free (CMF)-PBS and permeabilized with $0.1 \%$ Triton X-100 in CMF-PBS.

Image analysis. GFP-dSH2 and immunofluorescence analysis was performed using NIH Image J software (W. Rasband, National Institutes of Health, Bethesda, MD). Red-green-blue (RGB) three-time-point merged images and kymographs were constructed in NIH Image J. For immunocytochemical analysis, filopodial tips were scored as positive if the fluorescence intensity exceeded twice that observed in the central domain of the growth cone. For experiments examining percentages of protrusive or PY-positive filopodia and rates of filopodial initiation, all measurements were made from 5 min image sequences. Filopodial tips were scored as PY positive if they contained discrete GFP-dSH2 puncta three times greater than the average intensity of the growth cone center during this period. Filopodia were scored as motile if they exhibited any detectable length increases during the examination period. For experiments examining the effects of pharmacological inhibitors, analysis was performed starting $3 \mathrm{~min}$ after drug application. For BDNF experiments, analysis was performed beginning 5 min before BDNF addition and ending $10 \mathrm{~min}$ after. Rate of extension for filopodia was determined by measuring changes in length between images captured every 5 or $10 \mathrm{~s}$. Statistical significance was determined using Mann-Whitney $t$ test, and variance was reported as mean \pm SEM using InStat software (GraphPad Software, San Diego, CA). Immunoblot quantification was performed by measuring mean band intensities using NIH Image J analysis software.

Turning assay. All experiments involving local application of Src inhibitors were performed with a $40 \times$ air objective on a Zeiss (Oberkochen, Germany) Axiovert microscope equipped with a Coolsnap HQ CCD camera (Roper Scientific, Trenton, NJ). Micropipettes with a tip diameter of $1 \mu \mathrm{m}$ were filled with either $1 \times$ modified Ringer's culture medium or medium containing $50 \mu \mathrm{M}$ protein phosphatase 2 (PP2). Micropipettes were positioned at a distance of $30 \mu \mathrm{m}$ from the growth cone leading edge at $60-90^{\circ}$ with respect to the neurite axis. An electronically gated pressure application system (World Precision Instruments, Sarasota, FL) controlled by a programmable pulse generator (Master 8; A.M.P.I, Jerusalem, Israel) was used to generate $50 \mathrm{~ms}$ pulses with a positive pressure of $3 \mathrm{psi}$ at a frequency of $1 \mathrm{~Hz}$. Pulsatile ejection of an FITC-dextran solution during image acquisition confirmed production of a gradient similar to that used by Zheng et al. (1994). Using these and previous measurements, we estimate that the concentration gradient across a $10 \mu \mathrm{m}$ growth cone positioned $30 \mu \mathrm{m}$ from the micropipette tip to be $\sim 25-30 \%$. During each assay, images were collected using MetaMorph acquisition software (Universal Imaging Corporation, West Chester, PA) every $15 \mathrm{~s}$ for analysis of filopodial behavior. Only growth cones that extended at least $10 \mu \mathrm{m}$ during imaging were included in our analysis. Turning assays were stopped once axons extended $10 \mu \mathrm{s}$ or failed to advance $10 \mu \mathrm{m}$ within $60 \mathrm{~min}$. Turning angles were determined using MetaMorph software by measuring the angle of the $5 \mu \mathrm{m}$ distal segment of the neurite at the beginning and end of each trial.

Reagents and immunoblotting. BDNF (Promega, Madison, WI) and netrin-1 (R \& D Systems, Minneapolis, MN) were applied at concentrations of 100 and $200 \mathrm{ng} / \mathrm{ml}$, respectively. Herbimycin A (HA), PP2, PP3, SU66056, and SKI-1 were obtained from Calbiochem (La Jolla, CA). STI571 was a gift from Tony Koleske (Yale University, New Haven, CT). Antibodies used were as follows: $\beta 1$-integrin (Developmental Studies Hybridoma Bank, University of Iowa, Iowa City, IA), PY99 (Santa Cruz Biotechnology, Santa Cruz, CA), phospho-Src (Biosource International, Camarillo, CA), Cdc42 (PharMingen, San Diego, CA), and Alexa-Fluor conjugated $2^{\circ}$ antibodies (Molecular Probes). The polyclonal phosphoPAK antibody was provided by Jonathan Chernoff (Fox Chase Cancer Center). Immunoblotting for phospho-Src and phospho-PAK was performed as described previously (Robles et al., 2003), with the exception that four to five neural tubes were used for each lane, and dissected neural tubes were treated with inhibitors for $30 \mathrm{~min}$ before lysis. Alexa-546 phalloidin (Molecular Probes) was used to label filamentous actin (Factin). The biotinylated N-WASP Cdc42/Racl-binding (CRIB) peptide was synthesized by the University of Wisconsin Peptide Synthesis Facility as described previously (Vastrik et al., 1999), and internalization was confirmed by FITC-streptavidin staining.

\section{Results}

\section{PY imaging in live growth cones}

To investigate PY dynamics in motile growth cones, we used a fluorescent PY reporter construct consisting of tandem PYbinding SH2 domains fused to GFP (GFP-dSH2) (Fig. 1A,B) (Kirchner et al., 2003). To confirm the specificity of this indicator, we examined the degree of colocalization between GFP$\mathrm{dSH} 2$ fluorescence in live cells and PY-specific immunostaining in the same cells after fixation. Non-neuronal cells cultured from Xenopus neural tube exhibited enriched GFP-dSH2 labeling at focal adhesions, cell-substratum contacts known to contain concentrated tyrosine-phosphorylated proteins (Geiger et al., 2001), and the tips of some filopodia (Fig. 1C,D). Xenopus spinal neurons had similar enrichment of GFP-dSH2 fluorescence at the growth cone periphery and filopodial tips (Fig. $1 E-G$ ). The higher background fluorescence observed in GFP-dSH2expressing cells compared with PY antibody staining is likely attributable to unbound cytosolic GFP-dSH2. Ratiometric imaging of neurons double labeled with GFP-dSH2 and TMR-D demonstrated that GFP-dSH2 accumulations were independent of cell volume (Fig. $1 \mathrm{H}$ ). To confirm that the PY puncta we observe required tyrosine kinase activity, we treated growth cones with HA, a general inhibitor of tyrosine kinases. Acute HA application led to a rapid loss of PY enrichments, likely attributable to dephosphorylation by tyrosine phosphatases (Fig. 1I). Together, 
A

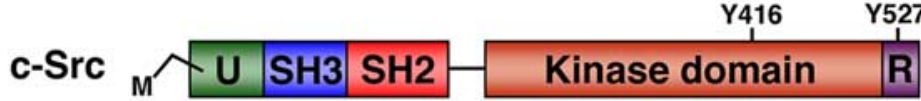

B

\section{\begin{tabular}{ll|l|l|}
\hline GFP-dSH2 & GFP & SH2 & SH2 \\
\cline { 2 - 3 }
\end{tabular}}
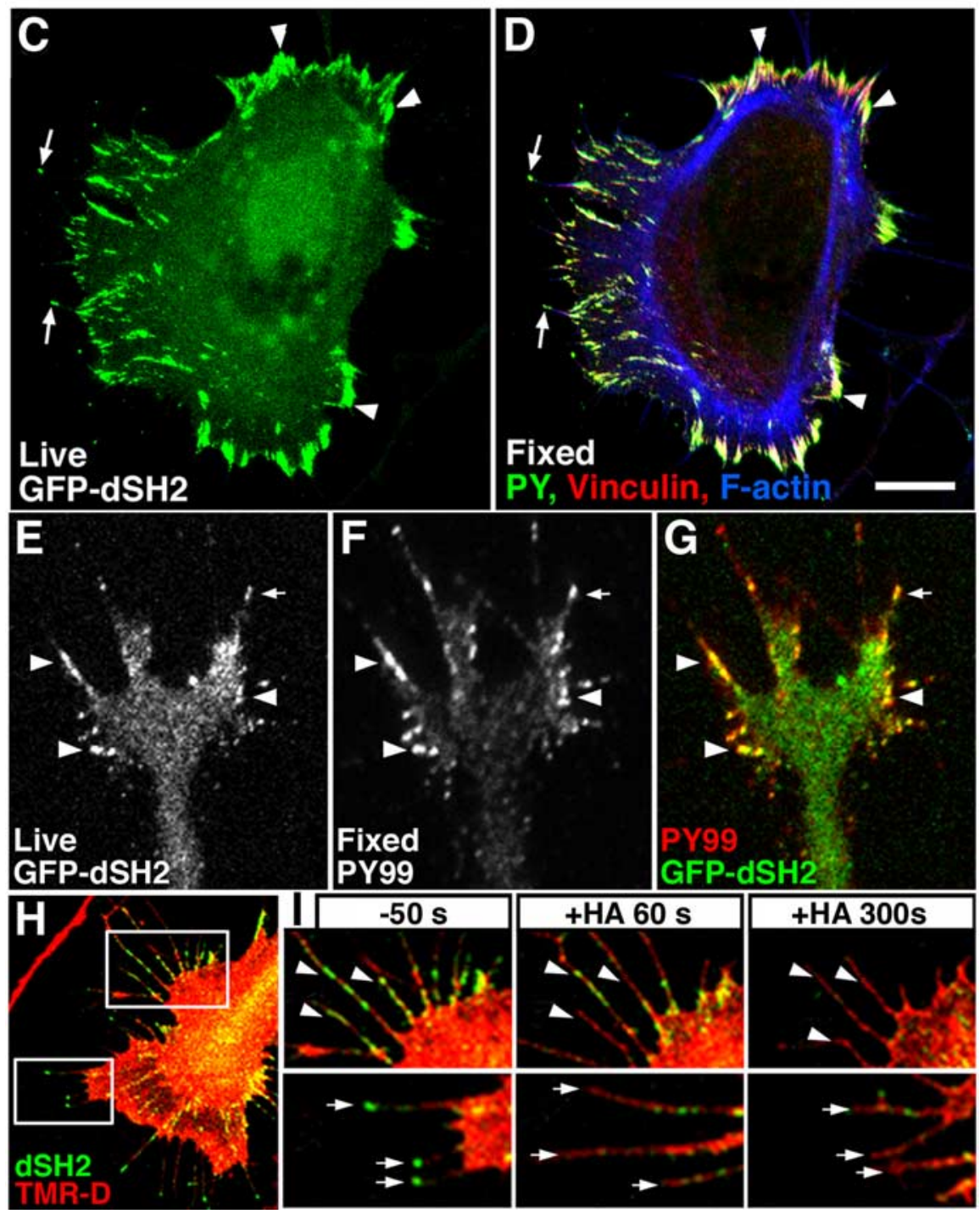

Figure 1. Imaging PY dynamics in living cells using GFP-dSH2. A, Structural domains of c-Src used in the construction of PY indicator (modified from Kirchner et al., 2003). M, Myristoylation sequence; $U$, unique region; $R$, regulatory domain. $B$, Schematic representation of the GFP-dSH2 construct. C, Live GFP-dSH2-expressing non-neuronal cell cultured from Xenopus neural tube. Note distinct focal adhesion labeling at cell periphery (arrowheads) and PY puncta at the tips of microspikes (arrows). D, The same cell fixed and triple labeled for PY (PY99; green), vinculin (red), and filamentous actin (blue). PY labeling in fixed cells is attributable entirely to immunofluorescent staining, because GFP-dSH2 fluorescence is lost during fixation. Note close correlation between live and fixed PY labeling (arrows) as well as colocalization with vinculin (arrowheads). Scale bar: $\boldsymbol{C}, \boldsymbol{D}, 10 \mu \mathrm{m} ; \boldsymbol{E}-\boldsymbol{H}, 5 \mu \mathrm{m}$. $\boldsymbol{E}$, Live growth cone on LN expressing GFP-dSH2. Note intense labeling in point contact structures within the central domain (arrowheads) and puncta in filopodial tips (arrows). $\boldsymbol{F}$, Growth cone from $\boldsymbol{E}$ fixed and immunolabeled with anti-phospho-tyrosine primary antibody (PY99). G, Merged image of live GFP-dSH2 and fixed PY99 fluorescence. Note that bright GFP-dSH2 puncta at the periphery colocalize with immunolabeled PY (arrows and arrowheads), whereas diffuse GFP fluorescence in the central domain shows no colocalization. $\boldsymbol{H}$, Merged images of a growth cone on LN expressing GFP-dSH2 (green) and labeled with TMR-D (red). $\boldsymbol{I}$, Time-lapse images during application of $10 \mu \mathrm{m}$ HA of boxed regions indicated in $\boldsymbol{H}$ at $2 \times$ magnification. Note that, before tyrosine kinase inhibition, many filopodia contain PY puncta at their tip (arrows) and point contacts near their base (arrowheads). At $60 \mathrm{~s}$ after HA treatment, PY is lost from the tips of filopodia (arrows) but remains in some point contacts (arrowheads). At 5 min after HA treatment, most PY clusters have dissolved, but several stable filopodia remain.

these findings confirm that GFP-dSH2 specifically localizes to PY-containing structures in growth cones.

We initially examined PY dynamics in growth cones on the extracellular matrix (ECM) protein $\mathrm{LN}$, which is known to activate focal adhesion kinase and Src tyrosine kinases during integrin receptor binding (Geiger et al., 2001). PY is concentrated primarily in two distinct subcellular locations in GFPdSH2-expressing growth cones on $\mathrm{LN}$, point contact structures resembling focal adhesions and filopodial tips (Fig. $2 A-C$ ) (supplemental movie 1, available at www.jneurosci.org as supplemental material). PY in point contacts was only observed in neurons cultured on LN and occurred predominantly in the central region of the growth cone and proximal regions of filopodia. Point contacts on LN formed and disassembled rapidly (average lifetime, $172.5 \pm 20.4 s ; n=28$ ), but their position remained stable with respect to the substratum, suggesting a role in adhesion (Fig. $2 D$ ). In contrast, PY puncta at the tips of filopodia had significantly shorter lifetimes than point contact PY (average lifetime, $85.1 \pm 5.6 \mathrm{~s} ; n=45 ; p<$ 0.0001 ), were highly motile, and often associated with elongating filopodia. In growth cones cultured on PDL, an artificial substratum that does not require receptor binding for cell adhesion, only tip PY was observed (Fig. $2 E-H$ ) and was often present at the tips of filopodia extending off the substratum surface (supplemental movie 3, available at www. jneurosci.org as supplemental material). Together, these findings suggest that integrin binding promotes the assembly of PYcontaining adhesion complexes, whereas $\mathrm{PY}$ at the tips of filopodia regulates protrusion independent of adhesion to ECM components.

Local Src kinase activity promotes filopodial initiation and extension

Filopodial extension is driven by the polymerization of parallel bundled actin filaments (Mallavarapu and Mitchison, 1999). However, the signals mediating this process are unknown. To determine whether PY at filopodial tips correlates with protrusion, we quantified the incidence of tip PY during three distinct filopodial behaviors: extension, stabilization, and retraction. Filopodial tips were scored as PY positive when their GFP-dSH2 fluorescence intensity was equal to or greater than the average intensity of the growth cone central domain. Given the large volume difference between the central domain and filopodia, this ensures that only filopodia with prominent PY enrichments are scored as PY positive. On both LN and PDL, filopodia exhibited the highest incidence of tip PY while extending and significantly lower incidence while stabilized or retracting (Fig. $2 \mathrm{~K}$ ). 
This positive correlation between filopodial extension and tip PY was observed in all growth cones examined and indicates that one or more tyrosine-phosphorylated proteins may promote the actinpolymerizing activity of the filopodial tip complex.

We demonstrated previously that inhibition of tyrosine kinase signaling in growth cones on LN leads to filopodial stabilization and lamellipodial collapse (Robles et al., 2003). The complex effects of tyrosine kinase inhibition on growth cones on LN may be attributable to the loss of PY at both filopodial tips and point contacts. To examine the signaling mechanisms mediating filopodial tip PY in the absence of adhesive point contacts, we examined growth cones cultured on PDL for all subsequent experiments. Our previous studies also suggested a specific role for Src family kinases in the regulation of filopodial motility. To directly examine the spatial patterns of Src kinase activity in growth cones on PDL, we immunofluorescently labeled growth cones with an antibody that specifically recognizes active Src family kinases autophosphorylated at Tyr418 (P-Src) (Ma et al., 2000). The specificity of this antibody in Xenopus was confirmed by Western blot of lysates from neural tubes treated with pharmacological inhibitors of tyrosine kinases and phosphatases (Fig. 3D). Whereas neurons on LN exhibit punctate P-Src labeling throughout the growth cone (Robles et al., 2003), the predominant site of P-Src enrichment in growth cones on PDL is at PY-

positive filopodial tips (Fig. $3 A-C$ ) (see Fig. $8 D$ ). Strong P-Src immunofluorescence was not detected in any of the PY-negative filopodial tips examined, likely attributable to recognition of P-Tyr418 by the PY antibody. However, a small percentage of PY-positive filopodia did not contain enriched levels of P-Src (Fig. 3A-C, arrowhead) (see Fig. $8 D$ ), suggesting that additional tyrosine-phosphorylated proteins localize to the tips of filopodia and may persist after inactivation or relocalization of Src kinases. The strong correlation of P-Src and PY immunofluorescence at the tips of filopodia suggests that local Src activity generates PY signals at the tips of filopodia.

The role of Src kinase activity in filopodial motility and PY signaling was examined using both general and Src-specific tyrosine kinase inhibitors. Filopodia were scored as PY positive or motile if they exhibited PY accumulations at their tips (as defined above) or detectable length increases during $5 \mathrm{~min}$ image sequences captured before and after drug treatment. The general tyrosine kinase inhibitor HA typically led to brief elongation of a subset of filopodia, followed by loss of tip PY and subsequent inhibition of filopodial protrusion. The majority of transiently elongating filopodia ( $84 \% ; n=25$ filopodia) maintained detectable PY enrichments during this period of rapid growth. The Src-specific inhibitors PP2, SU6656 (Fig. 3F), and SKI-1 (data not shown) had similar effects, strongly reducing the percentage of PY-positive and motile filopodia 3-8 min after drug applica-
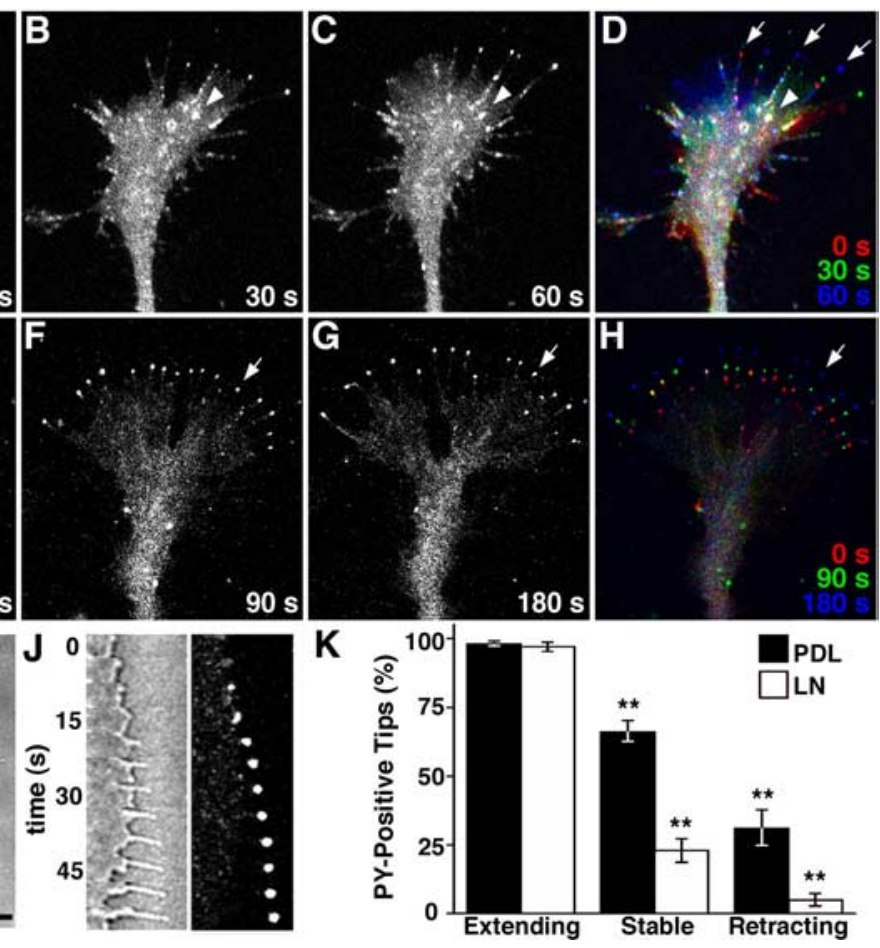

PDL

Figure 2. Tyrosine phosphorylation at the tips of filopodia correlates with filopodial protrusion. $\mathbf{A}-\mathbf{C}$, Time-lapse images at $30 \mathrm{~s}$ intervals of GFP-dSH2 fluorescence in a growth cone extending on LN. D, RGB three-time-point merge of this growth cone

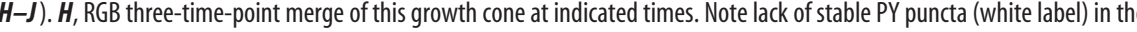
$J, 3 \mu \mathrm{m} . K$, Incidence of tip PY during periods of filopodial extension, stabilization, and retraction on both LN and PDL. $n>60$ filopodia in each condition. Data were obtained only from filopodia that exhibited all three behaviors. ${ }^{* *} p<0.001$.

tion. In contrast, inhibition of Abl tyrosine kinases with $3 \mu \mathrm{M}$ STI571 (Gleevec), a concentration that effectively disrupts hippocampal neuron dendrogenesis (Jones et al., 2004), did not reduce tip PY (data not shown). Although it is unclear why a subset of filopodia elongate in response to tyrosine kinase inhibition, this response is similar to the effects of attenuating retrograde flow by inhibiting myosin contractility (Lin et al., 1997). This suggests that Src kinases may regulate growth cone processes distinct from those occurring at filopodial tips, including those mediating retrograde flow or lamellipodial actin polymerization. However, the enrichment of active Src kinases at the tips of filopodia and the efficacy of Src-specific inhibitors in reducing filopodial motility during loss of tip PY both support a role for Src kinases at the tips of filopodia.

In addition to localizing to the tips of extending filopodia, PY often accumulated into puncta at the leading edge of growth cones before filopodial initiation. As shown in Figure $2 \mathrm{~J}$, a distinct cluster of PY formed at the future site of filopodial initiation and remained at the tip during extension of the nascent filopodium. This observation suggests that local tyrosine phosphorylation also regulates filopodial initiation. To determine whether Src kinase activity is specifically required for filopodial initiation, we compared rates of filopodium formation in the presence and absence of Src inhibitors. Acute PP2 application strongly reduced the frequency of filopodial initiation in growth cones cultured on 

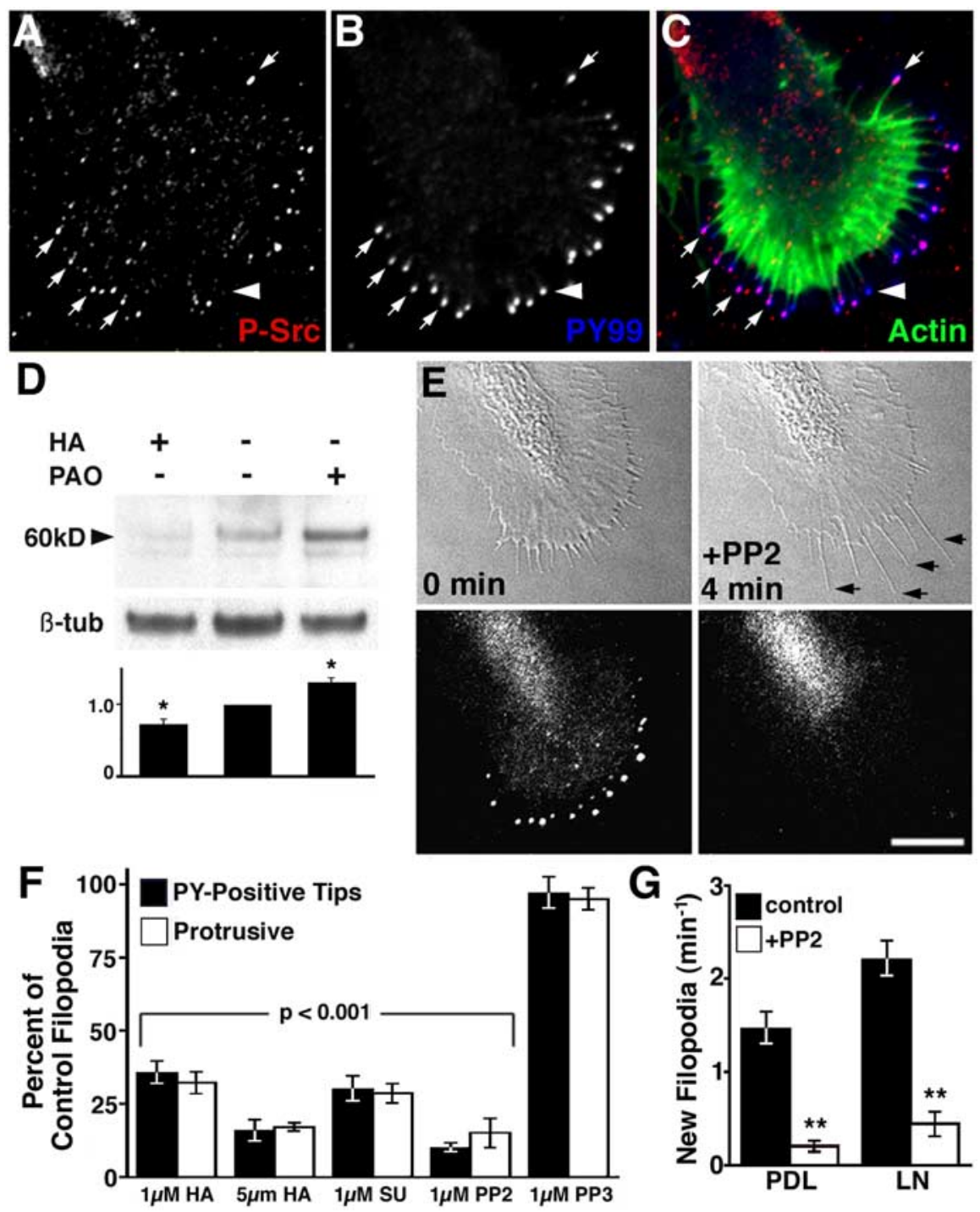

Figure 3. Src family kinase activity mediates tip PY and is required for filopodial initiation and elongation. $A, B$, Immunofluorescent staining of a growth cone on PDL for P-Src and PY. C, Merge of P-Src (red), PY99 (blue), and F-actin (green). Arrows indicate a subset of PY-positive filopodia with strong enrichment of P-Src. Arrowhead denotes a PY-positive filopodia lacking obvious enrichment in P-Src. D, Immunoblot for P-Src from Xenopus neural tube lysates incubated in control media or media containing either a tyrosine kinase inhibitor (20 $\mu \mathrm{MHA}$ ) or a tyrosine phosphatase inhibitor [100 $\mu \mathrm{m}$ phenylarsine oxide (PA0)] and then immunoblotted with anti-P-Src antibody. Note that the density of the band at $60 \mathrm{kDa}$, which represents one or more Src family kinases, was altered in response to pharmacological manipulations. This blot was reprobed with anti- $\beta$-tubulin ( $\beta$-tub) antibody to control for protein loading. Assay was repeated four times with similar results. Quantified band intensities are normalized to values for the control lane. $\boldsymbol{E}$, DIC and GFP-dSH2 fluorescence images of a growth cone on PDL before and after acute application of an Src-specific inhibitor (1 $\mu \mathrm{M}$ PP2). Immediately after Src inhibition, a subset of filopodia briefly lengthen during loss of PY (arrows), but all filopodia stop protruding after complete loss of PY. $\boldsymbol{F}$, The effects of tyrosine kinase inhibitors [1 and $5 \mu \mathrm{m} \mathrm{HA,} 1$ $\mu \mathrm{M}$ SU6656 (SU), $1 \mu \mathrm{M}$ PP2, and $1 \mu \mathrm{M}$ PP3, an inactive analog of PP2] on the number of PY-positive and protrusive filopodia, expressed as a percentage of the number before drug treatment. $n>8$ growth cones for each treatment. $\mathbf{G}$, Src inhibition reduces the rate of filopodial initiation on LN and PDL as measured during 5 min image sequences before and after drug application. $n>$ 10 growth cones for each condition. Scale bar: $\boldsymbol{A}-\boldsymbol{C}, 3 \mu \mathrm{m} ; \boldsymbol{E}, 5 \mu \mathrm{m} .{ }^{* *} p<0.001$.

both LN and PDL (Fig. 3G). Together, these findings suggest that Src-mediated phosphorylation is a necessary signal for the formation and extension of growth cone filopodia.

\section{Molecular perturbation of c-Src activity alters filopodial dynamics}

To confirm our pharmacological findings implicating Src family kinases in the regulation of filopodial protrusion, we examined the effects of expressing dominant-negative KD and CA mutants of c-Src. KD-Src competitively binds to c-Src substrates and in- terferes with their phosphorylation by endogenous Src kinases. The CA-Src mutant cannot be inhibited by Csk phosphorylation, which increases its kinase activity without altering substrate specificity (Worley et al., 1997). Expressing c-Src mutants in Xenopus embryos by RNA injection can result in both increased lethality and developmental abnormalities (Worley et al., 1997). To ensure proper early embryonic development, we injected a low concentration mRNA encoding mutant c-Src proteins into one or two blastomeres at the eight-cell stage. Using this approach, $<25 \%$ of the cells in the neural tube expressed the mutant proteins at detectable levels, and there were no visible effects on embryonic development.

Whereas high levels of KD-Src expression inhibited axonogenesis, lower expression levels allowed analysis of filopodial dynamics in neuronal growth cones with reduced Src kinase activity. KD-Src expression strongly altered growth cone morphology and motility. In extreme cases, growth cones had a simplified morphology, attributable in part to a marked reduction of filopodial protrusions (Fig. $4 B$ ). Immunocytochemical analysis revealed that $\mathrm{KD}$-Src expression significantly lowered the percentage of PY99positive filopodial tips per growth cone (Fig. 4D). Live imaging of KD-Srcexpressing neurons confirmed that the reduction in filopodial tip PY correlated with strong reductions in both the percentage of motile filopodia and the rate of filopodial initiation (Fig. $4 E, F$ ) (supplementalmovie2, availableatwww.jneurosci. org as supplemental material). Molecular perturbation of Src activity confirms our pharmacological data and supports a specific function for Src family kinases in the generation of local PY signals required for filopodial initiation and extension. However, these results do not exclude the possibility that multiple Src kinase family members phosphorylate targets at the tips of filopodia.

In contrast to the effects of KD-Src expression, growth cones expressing an active c-Src mutant often exhibited highly complex morphologies with many filopodial protrusions (Fig. 4C). Immunocytochemical analysis revealed that CA-Src expression significantly increased the percentage of PY99-positive filopodial tips per growth cone (Fig. 4D). Live imaging of CA-Src-expressing neurons confirmed that this increase in tip PY corresponded with an increase in the percentage of filopodia per growth cone that were motile (Fig. 4E) (supplemental movie 2, available at www.jneurosci.org as supplemental material). However, the most robust effect of CA-Src expression in neuronal growth cones was on the rate of filopodial initiation. CA-Src expression resulted in a more than twofold 
increase in the production of new filopodia (Fig. $4 F$ ). Together, these findings demonstrate that modulation of c-Src activity is sufficient to alter growth cone morphology and filopodial dynamics, further supporting a role for Src kinases within the filopodial tip complex.

\section{Regulation of filopodial PY by axon guidance cues}

Many chemotropic factors regulate axon outgrowth and guidance by altering filopodial motility, but the mechanisms underlying these effects have not been well characterized. To determine whether attractive guidance cues regulate motility by increasing filopodial PY levels, we imaged GFP-dSH2-expressing growth cones during stimulation with BDNF, which is known to positively affect the motility of Xenopus growth cones through TrkB activation (Ming et al., 1997). Initial experiments revealed that the effects of BDNF on growth cone motility was context dependent, in that responses were only detected in neurons that were not highly motile before stimulation (data not shown). Therefore, in these studies, we selected growth cones with few motile filopodia and low baseline PY levels before stimulation with guidance cues. Figure $5 A-D$ depicts a growth cone on PDL with many immotile filopodia lacking detectable PY accumulations before BDNF stimulation. Within 2 min of BDNF stimulation, this growth cone exhibited marked increases in filopodial number (Fig. $5 G$ ), motility, and tip PY (Fig. 5H). BDNF also rapidly stimulated the production of new PY-positive filopodia from the growth cone and axon shaft (Fig. 5E,F) (supplemental movie 3, available at www.jneurosci. org as supplemental material). On average, the number of filopodia peaked $8.6 \pm 0.8 \mathrm{~min}$ after BDNF stimulation (measured at 1 min intervals; $n=8$ growth cones). However, increased filopodial PY levels were also attributable to the accumulation of PY at the tips of preexisting filopodia. Detailed analysis of three growth cones with a total of 43 filopodia that were immotile and PY negative before BDNF application indicated that 40 of these filopodia acquired prominent PY accumulations at their tips within 15 min of BDNF stimulation. Subsequently, 39 of the 40 filopodia began to exhibit cycles of protrusion and retraction typical of motile filopodia on PDL (Wu and Goldberg, 1993) (supplemental movies 3, 4, available at www.jneurosci.org as supplemental material). Although there was a high degree of variability in the delay to filopodial PY accumulation, even within filopodia on the same growth cone, in every case filopodia that became motile after BDNF stimulation did so only after the accumulation of PY at their tip (Fig. 5I). Together, these results suggest that the increased filopodial initiation and motility stimulated by BDNF correlates with the accumulation of tyrosinephosphorylated proteins at their tips.

The percentage of filopodia with PY accumulations at their tips (measured at $1 \mathrm{~min}$ intervals) rapidly increased in response to BDNF and remained above prestimulation levels for up to 20 min (Fig. $5 H$ ), similar to the kinetics of BDNF-enhanced motility reported previously in Xenopus spinal and chick retinal growth cones (Ming et al., 1997; Gehler et al., 2004). However, this measurement does not account for the fact that motile filopodia typ- ically cycle through periods of high and low PY levels that correlate with periods of extension and retraction (Fig. 2). To quantify the effects of BDNF stimulation on PY within all filopodia, we calculated the percentage of growth cone filopodia that acquired at least one discrete PY accumulation at their tip (see criteria above) during a $5 \mathrm{~min}$ period before stimulation and 5-10 min after BDNF application. This interval was selected because a majority of preexisting filopodia acquire PY-positive tips within 10 min of BDNF application. Acute BDNF application to growth cones on PDL led to a significant increase in filopodial tip PY (Fig. $5 L$ ) that correlated with increased filopodial motility (data not shown) in $82 \%(n=17)$ of growth cones tested. This increased percentage of PY-positive filopodial tips was attributable to both the generation of new PY-containing filopodia (13.7 \pm 1.2 filopodia per growth cone before BDNF; $16.6 \pm 1.3$ filopodia per growth cone 5 min after BDNF; $p<0.05$ ), as well as the accumulation of PY and increased motility of existing filopodia. Importantly, BDNF-induced filopodial initiation, motility, and tip PY did not occur in response to heat-denatured BDNF or when Src kinases were blocked by simultaneous application of PP2 or SU6656 (Fig. 5L) (data not shown).

The fact that motile filopodia in the absence of BDNF have PY-positive tips suggests that this local signal is not exclusively downstream of TrkB activation but rather is a general signal associated with filopodial extension. To further support this possibility, we examined filopodial PY in response to netrin-1, a chemoattractant that promotes Xenopus growth cone motility through activation of the deleted in colorectal cancer (DCC) receptor (de la Torre et al., 1997). Netrin-1 increased tip PY and filopodial motility similar to BDNF (Fig. $5 J-L$ ) (data not shown), but only $48 \%$ of Xenopus spinal growth cones responded to netrin-1 $(n=23)$, complicating further examination of its effects on growth cone signaling. However, this finding confirms that PY accumulation at the tips of filopodia is a general signal associated with filopodial motility and not a specific signal initiated by BDNF activation of TrkB receptors. 

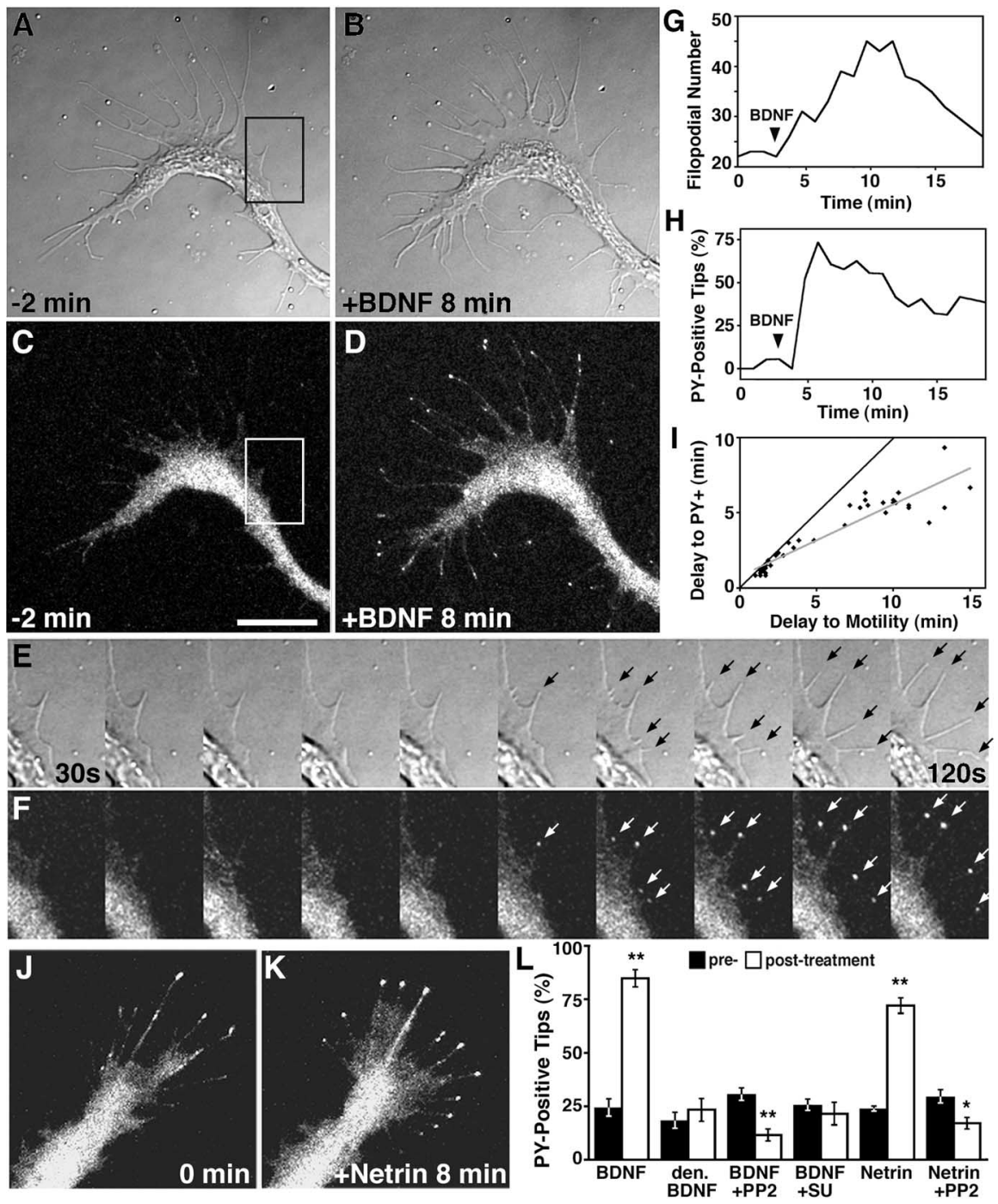

Figure 5. Modulation of filopodial tip PY by BDNF and netrin-1. A-D, Time-lapse DIC and GFP-dSH2 fluorescence images of a growth cone $2 \mathrm{~min}$ before and $8 \mathrm{~min}$ after BDNF application $(100 \mathrm{ng} / \mathrm{ml})$. Note that many filopodia lack enriched GFP-dSH2 at their tip before BDNF application but subsequently accumulate GFP-dSH2 after BDNF stimulation. $\boldsymbol{E}, \boldsymbol{F}$, Time-lapse DIC and GFP-dSH2 fluorescence images of boxed region indicated in $\boldsymbol{A}$ and $\boldsymbol{C} 30-120 \mathrm{~s}$ after BDNF application. Time between images is $10 \mathrm{~s}$. Arrows point to one preexisting filopodia that acquires tip PY $80 \mathrm{~s}$ after BDNF stimulation and three newly extended, PY-containing filopodia. $\boldsymbol{G}, \boldsymbol{H}$, Kinetics of increased filopodial initiation and GFP-dSH2 accumulation in response to BDNF in growth cone in $\boldsymbol{A}-\boldsymbol{D}$. I, Relationship between GFP-dSH2 accumulation and the initiation of motility in 39 filopodia (on 3 different growth cones) that were immotile and did not contain tip PY before BDNF application. The black line represents theoretical condition in which no delay separates PY accumulation and increased motility. Data points show actual delay, with the gray line representing the linear best fit for all data points. Note that the delay to motility initiation is disproportionately increased when the delay to PY accumulation is longer than $5 \mathrm{~min}$. $\boldsymbol{J}, \boldsymbol{K}$, Application of netrin- $1(200 \mathrm{ng} / \mathrm{ml})$ to growth cones with low baseline PY on PDL also increases filopodial tip PY. $L$, Percentage of PY-positive filopodia per growth cone before and after stimulation by guidance cues. BDNFinduced PY accumulation does not occur in response to denatured BDNF or during simultaneous Src inhibition with $1 \mu \mathrm{M}$ PP2 or 1 $\mu \mathrm{M}$ SU6656 (SU). Netrin effects are similarly blocked with $1 \mu \mathrm{m}$ PP2. $n>8$ growth cones for each data set. Scale bar: $\boldsymbol{A}-\boldsymbol{D}, \boldsymbol{J}, \boldsymbol{K}$, $5 \mu \mathrm{m} ; \boldsymbol{E}, \boldsymbol{F}, 3 \mu \mathrm{m} .{ }^{*} p<0.05 ;{ }^{* *} p<0.001$.

BDNF promotes growth cone motility by increasing levels of active Src kinases

The high degree of colocalization between PY99 and P-Src immunostaining at filopodial tips suggests that elevated PY correlates with an increase in active Src kinases at the tips of filopodia. To confirm this, we examined growth cone P-Src immunofluorescence in response to acute BDNF stimulation. Application of BDNF for $10 \mathrm{~min}$ before fixation resulted in a $49 \%$ increase in P-Src levels at the tips of filopodia (Fig. 6G), showing that increased filopodial PY in response to BDNF stimulation correlates with increased Src activity. Interestingly, $\mathrm{P}$-Src levels were also increased within the central domain of the growth cone, albeit to a lesser extent (19\% increase over control growth cones). Elevated P-Src at both locations was blocked by simultaneous application of the Src inhibitor PP2, confirming the specificity of our P-Src immunofluorescent staining.

BDNF has been shown previously to promote Xenopus spinal neuron outgrowth (Ming et al., 1997). To determine whether Src activity is specifically required for accelerated outgrowth, we examined the effects of Src inhibition on BDNFinduced neurite extension. Consistent with previous studies ( $\mathrm{Wu}$ and Goldberg, 1993), we find that the average growth rate for neurons on PDL is very slow, with many growth cones exhibiting no forward advance. However, bath application of BDNF significantly increased the average rate of neurite extension (Fig. $6 \mathrm{H}$ ), and this was blocked in a dose-dependent manner by simultaneous application of PP2, indicating that Src activity is required for the growth-promoting effects of BDNF. Although Src activity in the central domain of growth cones may contribute to the positive effects of BDNF, previous studies have correlated filopodial motility and neurite extension (Zheng et al., 1996). Therefore, it is likely that activation of Src kinases in filopodia contributes to the accelerated outgrowth by increasing PY signaling at filopodial tips.

\section{Asymmetric Src activity promotes growth cone turning}

Our observation that bath application of BDNF and netrin promotes growth cone motility by increasing filopodial tip PY suggests that growth cone pathfinding behaviors in vivo may be controlled through local modulation of filopodial PY levels. Within this model, the direction of axon extension would be determined by the distribution of motile, PY-containing filopodia at the growth cone periphery. This possibility is consistent with studies demonstrating that chemotropic growth cone turning coincides with asymmetric filopodial distribution toward the source of a diffusible chemoattractant (Zheng et al., 1996). To test whether asymmetric tyrosine phosphorylation is sufficient to promote axon turning, we locally applied the Src inhibitor PP2 from a micropipette using the method described by Zheng et al. (1994). However, to expose growth cones to a sharper PP2 concentration gradient, we positioned the micropipette $30 \mu \mathrm{m}$ from the growth cone center. Using our own and previous measurements (Zheng et al., 1994), we estimate that, under these conditions, the concentration gradient across a distance of $10 \mu \mathrm{m}$ is $\sim 25-30 \%$, with the highest concentration at the growth cone being 50 - to 100 - 
fold less than that in the micropipette. Local pulsatile application of media containing $50 \mu \mathrm{M}$ PP2 for $5 \mathrm{~min}$ resulted in a significant asymmetry in filopodial tip PY levels as determined by PY99 immunocytochemistry (Fig. 7A,B). The tips of filopodia on the side of the growth cone nearest the micropipette exhibited nearly a threefold decrease in PY levels compared with those on the far side of the growth cone $(359.4 \pm 94.3$ vs $1070.4 \pm 144.4$ arbitrary units; $p<0.001 ; n=49$ filopodia on 3 growth cones). Conversely, no significant asymmetry in PY levels was observed in control growth cones exposed to a $5 \mathrm{~min}$ local application of culture media $(1018.63 \pm 215.4$ vs $1032.9 \pm 175.5$ arbitrary units; $p=0.744 ; n=31$ filopodia on 3 growth cones).

To determine whether locally reduced Src kinase activity can influence the direction of neurite outgrowth over time, we performed turning assays as described previously (Zheng et al., 1994, 1996). Local application of PP2 caused the majority of growth cones examined to stall or collapse (15 of 25). However, most of the growth cones that continued to extend exhibited repulsive turning away from the source of PP2 (Fig. 7E-G). The average turning angle of these growth cones was significantly greater than that of growth cones exposed to local pulsatile ejection of culture media as a control (Fig. $7 C, D, G)(p<0.001 ; n=$ 10 growth cones for each condition). To determine whether turning correlated with an asymmetry in filopodial dynamics, we examined the rate of filopodial initiation on both sides of the growth cone. Local PP2 application resulted in significantly more filopodia initiated on the side farthest from the source of the Src inhibitor compared with the side nearest the micropipette (Fig. $7 H$ ). These results indicate that the direction of neurite outgrowth can be controlled by relative levels of Src activity and suggest that guidance cues may regulate growth cone pathfinding by locally modulating filopodial PY levels.

\section{Cdc42 activity promotes tyrosine phosphorylation at the filopodial tip}

The chemotropic effects of BDNF on growth cones have been linked to Cdc42 activation (Yuan et al., 2003). However, the localization of Cdc42 in growth cones and its relationship to PY signaling is unknown. Immunofluorescent staining demonstrated that Cdc42 is located throughout the growth cone but is specifically enriched at the tips of PY-positive filopodia (Fig. $8 \mathrm{~A}-$ $D)$, suggesting that Cdc42 is associated with filopodial PY and extension. To determine whether $\mathrm{Cdc} 42$ activity is required for Src-dependent tyrosine phosphorylation at filopodial tips, we reduced Cdc42 activity with toxin B, a general Rho GTPase inhibitor, or by expressing DN Cdc42 in a subset of spinal neurons. Both treatments significantly reduced the percentage of PY-
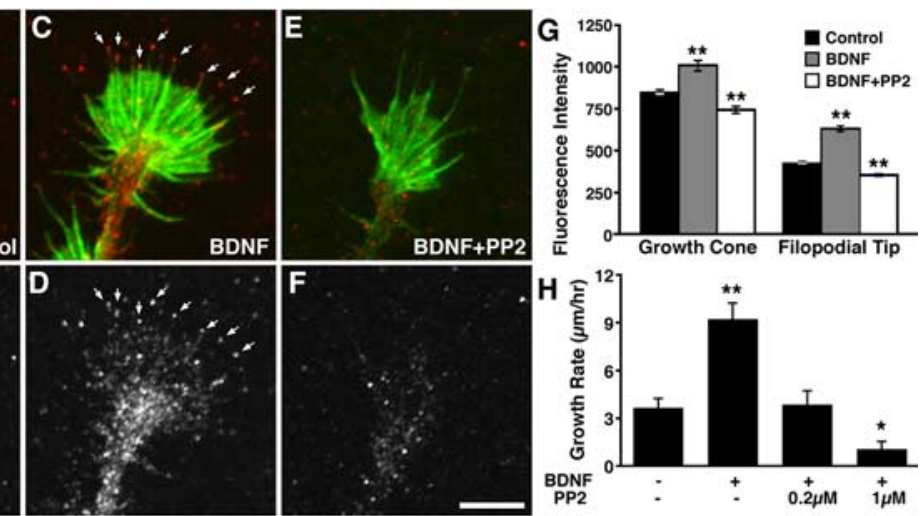

Figure 6. BDNF increases Src activity in growth cones. $\boldsymbol{A}-\boldsymbol{F}$, Single-channel fluorescence $(\boldsymbol{B}, \boldsymbol{D}, \boldsymbol{F})$ and merged images $(\boldsymbol{A}, \boldsymbol{C}, \boldsymbol{E})$ of P-Src and phalloidin staining in control $(\boldsymbol{A}, \boldsymbol{B})$, BDNF-treated $(\boldsymbol{C}, \boldsymbol{D})$, and BDNF plus PP2-treated $(\boldsymbol{E}, \boldsymbol{F})$ growth cones. Note
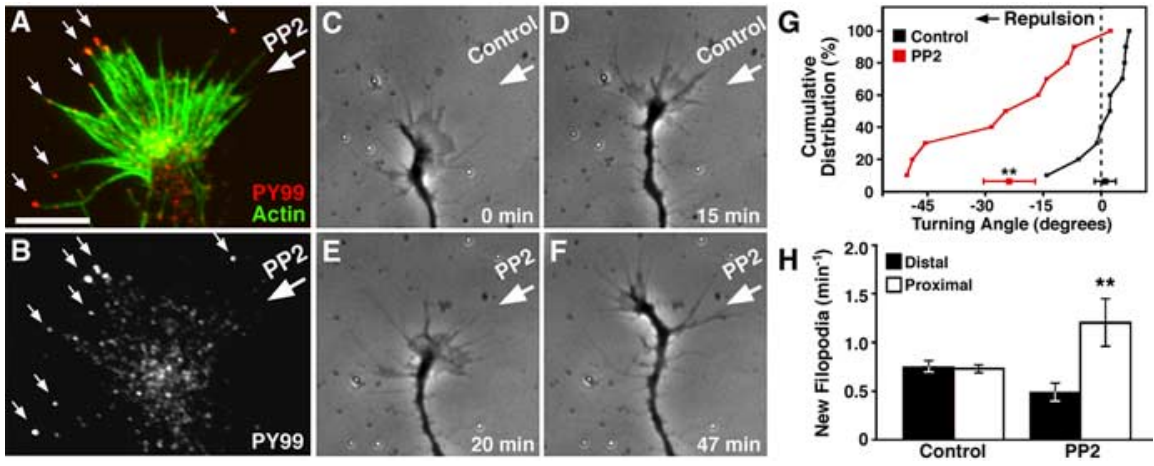

Figure 7. Local Src inhibition stimulates repulsive growth cone turning. $\boldsymbol{A}, \boldsymbol{B}$, Single-channel and merged images of PY99 immunofluorescence and phalloidin staining of a growth cone exposed to a gradient of PP2 for 5 min. Note that the number of PY-positive filopodia (small arrows) is reduced on the side of the growth cone closest to the source of PP2 (indicated by large arrow tionately generated on the distal side of the growth cone. Scale bar: $\boldsymbol{A}, \boldsymbol{B}, 5 \mu \mathrm{m} ; \boldsymbol{C}-\boldsymbol{F}, 15 \mu \mathrm{m}$. ${ }^{* *} p<0.001$.

positive filopodia per growth cone as determined by PY immunostaining (Fig. 8 E). To confirm these results in live cells, we used a cell-permeable Cdc42-specific inhibitory peptide composed of the CRIB domain of N-WASP fused to the Antennapedia internalization sequence (Vastrik et al., 1999). Acute application of the CRIB peptide partially reduced baseline tip PY, whereas pretreatment with the peptide for 5 min prevented BDNF-induced increases in filopodial PY and motility (Fig. $8 F$ ). Consistent with previous reports, we also find that expression of DN Cdc42 blocks BDNF-induced filopodial initiation and motility (see Fig. 10C). These data indicate that Cdc42 activity is required for Srcdependent tyrosine phosphorylation at the tips of filopodia. However, expression of CA-Cdc42 alone did not increase the percentage of PY-positive filopodial tips (Fig. 8E), suggesting that Cdc42 activity is necessary but not sufficient to promote filopodial tip PY. Together, these findings support a model in which modulation of $\mathrm{Cdc} 42$ activity regulates tyrosine phosphorylation at the tips of filopodia. 

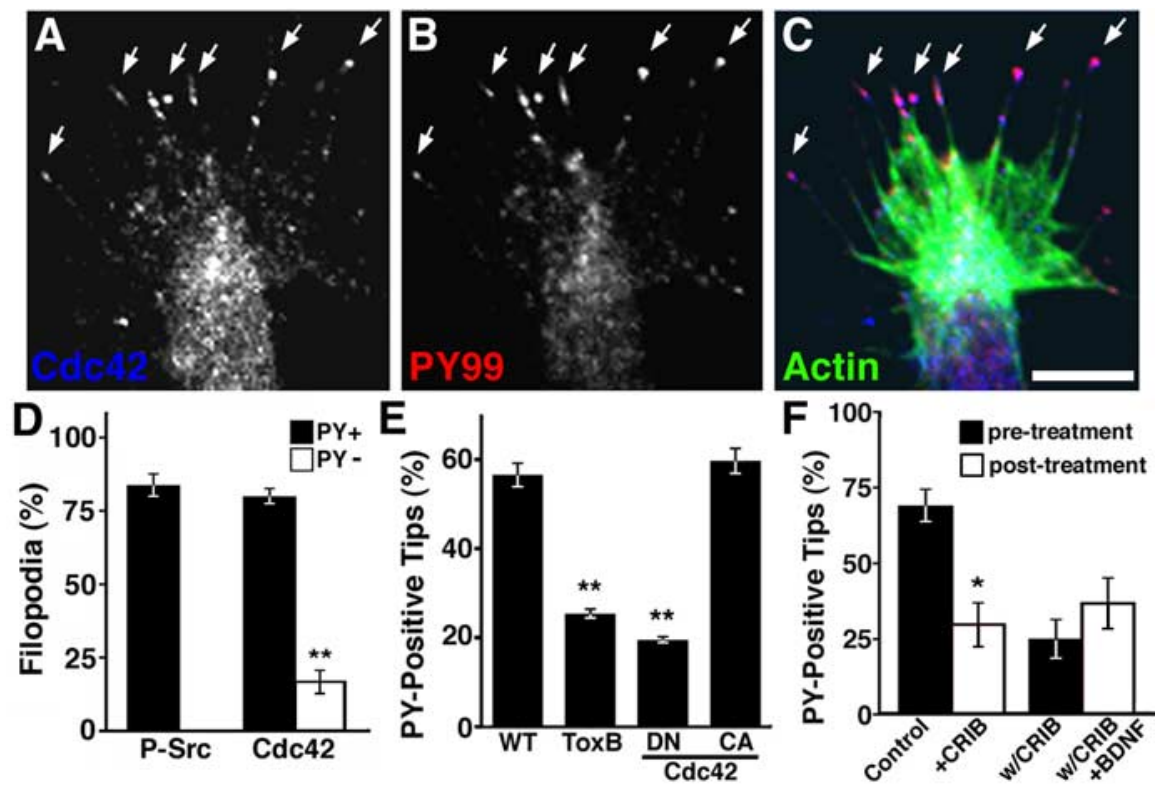

Figure 8. Cdc42 localizes to filopodia and regulates tip PY. $\boldsymbol{A}, \boldsymbol{B}$, Immunofluorescent staining for $(\mathrm{d} c 42$ and PY. $\boldsymbol{C}$, Merge of channels in $\boldsymbol{A}$ and $\boldsymbol{B}$ with F-actin staining in green. Note high degree of $\mathrm{Cd}(42$ and PY colocalization (magenta) at the tips of filopodia (arrows). D, Quantification of P-Src and Cdc42 colocalization to PY-positive and -negative filopodia. P-Src accumulations were not observed in PY-negative filopodia. $\boldsymbol{E}$, The percentage of PY-positive filopodia per growth cone determined by PY 99 immunolabeling shows that PY at filopodial tips is regulated by $C$ dc 42 activity. Inhibition of all Rho GTPases with toxin B (ToxB) or $\mathrm{Cd} 42$ selectively by expression of dominant-negative $\mathrm{Cd}(42$ reduces the percentage of PY-positive tips. Expression of a constitutively active mutant Cdc42 does not significantly alter filopodial tip PY. WT, Wild type. F, Direct inhibition of Cdc42 by application of CRIB peptide reduces tip PY in dSH2-expressing growth cones with high initial baseline PY. Pretreatment with CRIB peptide also blocks BDNF-induced increases in tip PY. $n>8$ growth cones for each data set. Scale bar, $5 \mu \mathrm{m}$. ${ }^{*} p<0.05$; ${ }^{* *} p<0.001$.

\section{PAK localizes to filopodial tips and regulates filopodial motility}

Recent studies have identified PAK and N-WASP as cytoskeletal regulators that are synergistically activated by Src kinases and Cdc42 (Renkema et al., 2002; Torres and Rosen, 2003). Using both immunofluorescent staining and GFP-N-WASP expression, we find that N-WASP does not localize to the tips of growth cone filopodia (data not shown). N-WASP may be a target of Src phosphorylation localized within the growth cone central domain, in which it may promote branched nucleation of actin filaments from preexisting filaments via the Arp $2 / 3$ complex (Millard et al., 2004). In contrast, a DsRed-tagged PAK1 protein was strongly enriched at the tips of filopodia (Fig. 9A,B), consistent with the reported localization of active PAK to the leading edge of migrating fibroblasts (Sells et al., 2000). Analysis of filopodial behaviors in DsRed-PAK-expressing neurons showed that PAK accumulation occurs predominantly at the tips of extending filopodia (Fig. 9C). To compare the spatial dynamics of PAK and GFP-dSH2 localization, we coexpressed DsRed-PAK and GFP$\mathrm{dSH} 2$ in Xenopus growth cones. Strikingly, DsRed-PAK and GFP-dSH2 precisely colocalize to the tips of extending filopodia (Fig. 9D-G) (supplemental movie 4, available at www.jneurosci. org as supplemental material). Kymography confirmed that both signals concentrate at the filopodial tip during extension and dissipate during filopodial retraction (Fig. 9G). The close correlation between PAK and PY puncta at the tips of extending filopodia is consistent with the possibility that PAK is tyrosine phosphorylated at the tips of growth cone filopodia. PAK enrichment was also disrupted by acute Src inhibition (Fig. $9 H, I$ ), with the majority of the transiently elongating filopodia $(76.5 \% ; n=17$ filopodia) maintaining enriched PAK at their tip during the brief period of rapid growth. This suggests that PAK localization to filopodia requires Src activity and that the sustained inhibition of filopodial elongation observed in response to Src inhibition may be attributable to the dissociation of PAK from the filopodial tip.

Tyrosine phosphorylation of PAK by Src kinases has been shown to enhance PAK activity after Cdc42-dependent destabilization of its inhibitory dimerization (Renkema et al., 2002). To determine whether PAK activity in Xenopus neurons is regulated by $\mathrm{Cdc} 42$ and Src kinases, we immunoblotted neural tube lysates with an antibody that specifically recognizes active PAK kinases autophosphorylated at Thr423 (Sells et al., 2000). This antibody is specific to a region of human PAK1 that is conserved in the Xenopus homolog XPAK1 and the closely related XPAK3, which is enriched in the nervous system during periods of axon outgrowth (Souopgui et al., 2002). Western blots show that this phospho-PAK antibody recognizes a single band at $\sim 62 \mathrm{kDa}$, which is the expected size of xPAK1 and xPAK3 (Fig. 9J). The need to restrict the expression of mutant $\mathrm{Src}$ and Cdc42 proteins to a small number of cells in the neural tube precluded analysis of their effects on phospho-PAK levels by immunoblot. However, levels of phospho-PAK in the neural tube were reduced by application of Src inhibitors (PP2 and SU6656) or the N-WASP CRIB peptide. This indicates that Src and Cdc42 regulate PAK activity in the Xenopus neural tube.

We have shown that $\mathrm{Src}$ and Cdc42 are necessary downstream signals for enhanced filopodial initiation and motility by BDNF (Figs. $5 L, 8 F$ ). To determine whether PAK activity is required for the growth-stimulating effects of BDNF, we examined growth cones expressing DN-PAK1, which contains mutations that disrupt both kinase activity and GTPase binding (Kiosses et al., 1999). Because of the high degree of sequence identity between PAK1 and PAK3 (79\% overall and 97\% in the catalytic domain) and their similar regulation (Chong et al., 2001), this DN protein is expected to interfere with the function of both PAK1 and PAK3 (J. Chernoff, personal communication). We find that expression of DN-PAK1 blocked BDNF-induced increases in filopodial number (data not shown) and motility (Fig. 10C). The inhibitory effects DN-PAK were similar to the effects of DN-Cdc42, suggesting that these proteins are components of a common signaling pathway downstream of BDNF. Although both Rac1 and Cdc42 can activate PAK, Racl is not enriched at the tips of growth cone filopodia (data not shown), suggesting that Cdc42 exclusively activates PAK at the filopodial tip. Together, our findings indicate that PAK activity is required for BDNF-induced filopodial dynamics downstream of Src and Cdc42 signaling.

To determine whether filopodial motility and PY signals are regulated by PAK activity in the absence of exogenous stimulation, we examined the effects of expressing DN and CA PAK1 mutant proteins. CA-PAK1 is a catalytically active mutant deficient in GTPase binding and unable to form autoinhibitory homodimers. Growth cones expressing DN-PAK had a significantly lower percentage of PY-positive filopodia as determined by PY99 immunostaining (Fig. 10D), suggesting that PAK activity downstream of Cdc42 is necessary for either filopodial tip complex 
formation or the localization of additional tyrosine-phosphorylated proteins. Similar to the effects of CA-Cdc42 (Fig. 8 F), expression of CA-PAK did not significantly increase filopodial PY (Fig. 10D). However, unlike CA-Cdc42-expressing growth cones, CA-PAK-expressing growth cones are more complex and often distinguished by long, branched filopodia (Fig. 10B). Live imaging of growth cones expressing CA-PAK revealed that enhanced PAK activity causes a robust increase in filopodial initiation (Fig. 10E), similar to the effects of CA-Src expression (Fig. 4F). Conversely, DN-PAK expression resulted in simpler growth cones (Fig. 10A) and a $52 \%$ decrease in filopodial initiation (Fig. $10 \mathrm{E})$, which is also similar to the effects of KD-Src expression (Fig. 4E). Interestingly, CA-PAK expression also strongly increased the rate of filopodial extension, whereas DN-PAK decreased this rate (Fig. $10 F)$. These results suggest that PAK is a component of the filopodial tip complex and functions to promote the initiation and extension of growth cone filopodia downstream of Src and Cdc42 signals.

\section{Discussion}

Our findings demonstrate that spatially and temporally precise patterns of Src kinase activity regulate filopodial dynamics. Live imaging of tyrosine phosphorylation suggests that PY at the tips of filopodia promotes filopodial formation and motility. Active Src kinases colocalize with Cdc42 at the tips of extending filopodia, suggesting that synergistic activation of their downstream targets may regulate the actin-polymerizing activity of the filopodial tip complex. We identified the Ser/ Thr kinase PAK as a component of the tip complex that may transduce Src and Cdc42 signals into increased actin polymerization. Together, our data support a model in which local tyrosine phosphorylation promotes neurite growth and guidance by cooperatively activating Cdc42 effectors.

Live imaging of PY dynamics in motile growth cones allowed us to characterize two distinct structures containing tyrosinephosphorylated proteins in growth cones on LN: stable point contacts and motile filopodial tips. Point contacts likely mediate adhesion to the ECM, whereas filopodial tip PY regulates protrusion independent of ECM interactions. PY at the tips of filopodia had been proposed previously to inhibit filopodial extension (Wu and Goldberg, 1993). However, by correlating PY dynamics directly with motility in live growth cones, we find that PY at filopodial tips promotes extension. This conclusion is based on the following results: (1) tip PY is predominantly associated with extending filopodia, (2) tyrosine kinase inhibition strongly reduces tip PY as well as filopodial initiation and protrusion, and (3) guidance cues that increase filopodial motility correspondingly increase the percentage of PY-positive filopodial tips. The discrepancy between these findings and previous work is likely
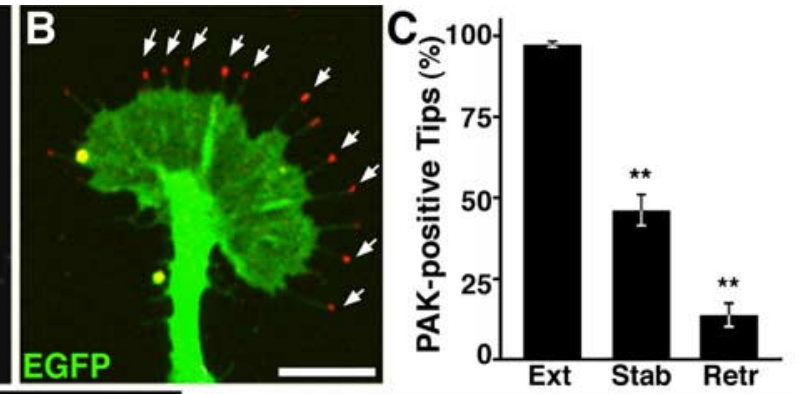
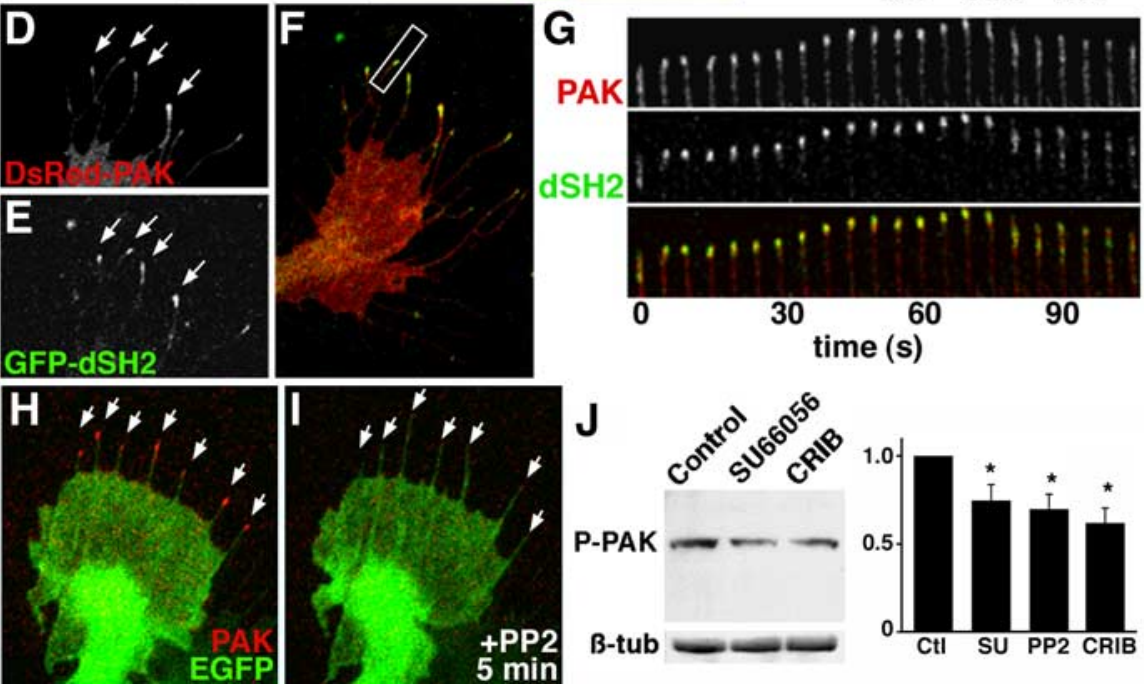

B-tub CtI SU PP2 CRIB
Figure 9. PAK colocalizes with PY at the tips of extending filopodia. $\boldsymbol{A}, \boldsymbol{B}$, Fluorescence images of a growth cone expressing DsRed-PAK1 and GFP. Note robust enrichment of PAK at the tips of filopodia (arrows in $\boldsymbol{B}$ ). $\boldsymbol{C}$, Incidence of DsRed-PAK accumulation PaKand condition. $\boldsymbol{D}-\boldsymbol{F}$, Individual fluorescence channels and merged image $(\boldsymbol{F})$ of a growth cone coexpressing DsRedPY during filopodial extension ( $t=15-45$ s) and de mage of a growth cone expressing DsRed-PAK and GFP before and after Src inhibition (1 $\mu \mathrm{M}$ PP2). Note the DsRed-PAK accumuloading, and the assay was repeated three times with similar results. Separate trials demonstrated similar results for the $\mathrm{SrC}$ inhibitor PP2. Quantified P-PAK band intensities for all experiments were normalized to control lane (Ctl). Scale bar: $\boldsymbol{A}, \boldsymbol{B}, \boldsymbol{D}-\boldsymbol{F}, \boldsymbol{H}$, I, $5 \mu \mathrm{m} ; \mathbf{G}, 2.5 \mu \mathrm{m} .{ }^{*} p<0.05 ;{ }^{* *} p<0.001$.

attributable to the difficulty in correlating filopodial behavior with PY signaling by immunofluorescence analysis.

Our data indicate that phosphorylation by Src family kinases is responsible for the PY accumulations detected with the $\mathrm{dSH} 2$ GFP indicator. This is suggested by the high degree of colocalization between PY and active Src kinases at the tips of filopodia, as well as by the efficacy of multiple Src-specific inhibitors in rapidly reducing filopodial PY levels. Furthermore, directly altering Src kinase activity in growth cones by expressing active and interfering mutants of the Src family kinase c-Src is sufficient to alter filopodial formation, extension, and PY signaling. Because many different receptor types have been shown to regulate Src kinase activity (Thomas and Brugge, 1997), it is possible that guidance cue receptors themselves directly regulate Src kinases. In fact, the Src family kinase Fyn has been shown to bind to the intracellular domains of TrkB, EphA, and plexin-A2 receptors (Iwasaki et al., 1998; Sasaki et al., 2002; Knoll and Drescher, 2004). More recently, Src kinases were found to bind and directly phosphorylate the netrin receptor DCC in response to netrin stimulation ( $\mathrm{Li}$ et 

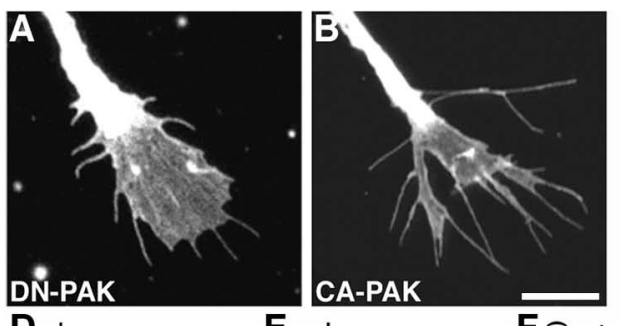

\section{C}
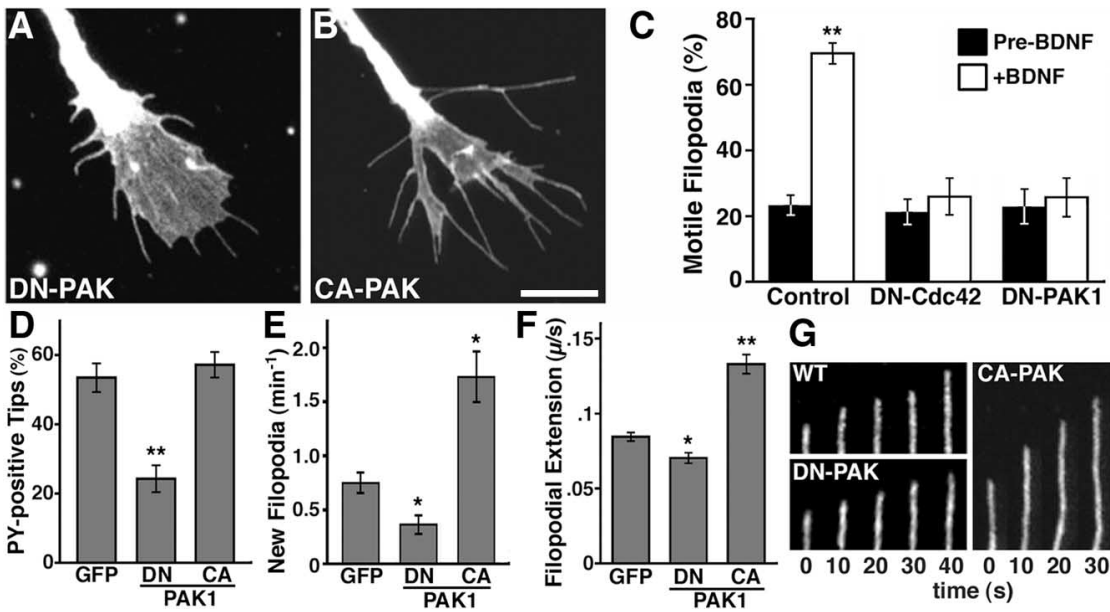

G

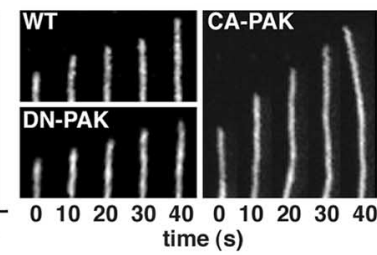

Figure 10. PAK activity regulates filopodial motility. $A, B$, Fluorescence images of growth cones on PDL coexpressing DN-PAK or CA-PAK together with GFP. C, Stimulation of filopodial motility by BDNF is blocked by expression of either DN-Cdc42 or DN-PAK. $n>12$ growth cones for each condition. $\boldsymbol{D}-\boldsymbol{F}$, Effects of DN-PAK or CA-PAK expression on the percentage of PY-positive tips $(\boldsymbol{D})$, the rate of filopodial initiation $(\boldsymbol{E})$, and the rate of filopodial extension $(\boldsymbol{F}) . n>20$ growth cones for each condition. $\boldsymbol{G}$, Examples of extending filopodia from growth cones with varying levels of PAK activity. The rate of extension is increased in a CA-PAKexpressing filopodium compared with wild type (WT). Scale bar: $A, B, 5 \mu \mathrm{m} ; \mathbf{G}, 2 \mu \mathrm{m}$. ${ }^{*} p<0.05 ;{ }^{* *} p<0.001$.

al., 2004; Liu et al., 2004; Meriane et al., 2004; Ren et al., 2004). However, it is likely that the Src-dependent signals we observe at the tips of filopodia are attributable to additional tyrosinephosphorylated proteins, because receptors are typically more spatially diffuse (Guirland et al., 2004) and therefore these PY signals may be undetectable by GFP-dSH2 imaging. Our findings indicate that BDNF stimulation increases levels of active Src kinases at the tips of filopodia and within the growth cone central domain. Although it is unknown whether Src family kinases directly interact with TrkB receptors in Xenopus spinal neurons, our data indicate that Src activation is a requisite downstream signal for BDNF-stimulated growth cone motility and outgrowth.

Modulation of filopodial PY signaling by guidance cues suggests that local regulation of filopodial PY and motility may underlie growth cone pathfinding behaviors. This possibility is consistent with the postulated role for filopodia as sensory protrusions that probe the extracellular environment for instructive cues. We demonstrated that local application of a Src-specific inhibitor to one side of the growth cone results in an asymmetric distribution of PY-containing filopodia. Functionally, local Src inhibition led to the disproportionate formation of new filopodia and repulsive growth cone turning. Many studies have shown that local application of BDNF or netrin can stimulate attractive growth cone turning of cultured Xenopus spinal neurons, and more recently turning toward netrin was found to require Src activity (Li et al., 2004; Meriane et al., 2004). Based on our findings that Src activity is required for the changes in filopodial dynamics elicited by BDNF and netrin, it is likely that local changes in PY signaling are involved in chemotropic responses toward these factors. Importantly, we show for the first time here that disproportionate Src activity is sufficient to promote growth cone turning (Fig. 7). In addition, because Cdc42 localizes to PY-positive filopodial tips and modulates PY signaling, it is also likely that active $\mathrm{Cdc} 42$ is asymmetrically distributed among filopodia during chemotropic turning. This possibility is supported by molecular perturbation studies showing that $\mathrm{Cdc} 42$ activity is necessary for chemotropic turning stimulated by BDNF (Yuan et al., 2003).

In this study, we implicate PAK as a downstream target of both
Src and Cdc42 at the tips of growth cone filopodia. In addition to robust enrichment of PAK at the tips of PY-positive filopodia during extension, expression of an interfering PAK mutant blocked BDNFinduced increases in filopodial motility, similar to the effects of Cdc42 inhibition. This suggests that PAK serves as an effector for Cdc42 in the regulation of filopodial PY signaling. PAK activation by $\mathrm{Cdc} 42$ is thought to occur through a direct interaction that releases PAK from autoinhibitory dimerization (Bokoch, 2003). This conformational change exposes a target tyrosine residue for Src phosphorylation (Renkema et al., 2002), which greatly increases its kinase activity compared with Cdc42 binding alone. Therefore, we propose that active $\mathrm{Cdc} 42$ at the tips of filopodia promotes tyrosine phosphorylation by increasing the availability of Src targets such as PAK. The decreased percentage of PY-positive filopodia observed in growth cones expressing either DN-Cdc42 or a DN-PAK mutant deficient in GTPase binding is consistent with a reduction in the availability of PAK as a target for Src phosphorylation. Although it is likely that additional Src targets localize to the tips of filopodia, the effects of reduced PAK activity on filopodial PY levels suggest that PAK activity may be required for the localization and/or activation of these proteins. However, expression of CA-Cdc42 or CA-PAK did not increase tip PY, indicating that increased target availability alone is insufficient to increase filopodial PY signaling. In fact, the bidirectional modulation of filopodial PY levels by active and interfering c-Src mutants indicates that the absolute level of Src kinase activity may be a more critical regulator of PY signaling in filopodia.

PAK1-PAK3 are highly conserved serine/threonine kinases that regulate actin dynamics through direct phosphorylation of several downstream targets. Live imaging of growth cones expressing mutant PAK proteins indicated that PAK regulates filopodial dynamics during neurite outgrowth. Filopodial extension is driven by actin polymerization at the distal ends of filopodial actin filaments (Mallavarapu and Mitchison, 1999). Both PAK localization and regulation of filopodial dynamics suggest that PAK promotes actin polymerization in filopodia. However, it is unclear what downstream PAK substrate may mediate these effects. Both myosin light chain kinase and the regulatory light chain of myosin II are inactivated through phosphorylation by PAK (Bokoch, 2003). However, the presence of parallel bundled actin filaments in distal filopodia suggests that conventional myosins do not function in filopodial extension. PAK has also been shown to phosphorylate myosin VI in vitro (Buss et al., 1998). Although a function for this unconventional myosin in growth cones has not been demonstrated, myosin $\mathrm{V}$ activity has been shown to promote filopodial extension (Wang et al., 1996). These findings identify unconventional myosins as potential PAK targets within filopodia. Although we cannot exclude involvement of yet unidentified PAK targets, PAK is also known to phosphorylate and activate LIM kinases, which promote actin assembly by phosphorylating and inhibiting the actin severing/depolymerizing activity of actin-depolymerizing factor (ADF)/cofilin. Therefore, local PAK activity at the tips of filopodia could serve to prevent actin disassembly and thereby promote actin polymer- 
ization and filopodial protrusion. Inactivation and dissociation of PAK from the tips of filopodia may serve to stop actin polymerization through disinhibition of the actin severing/depolymerizing activity of $\mathrm{ADF} /$ cofilin, leading to filopodial retraction.

Growth cone pathfinding is thought to require integration of multiple intracellular signals stimulated by extracellular guidance cues. Our findings demonstrate that spatially and temporally regulated patterns of tyrosine phosphorylation control filopodial behavior downstream of guidance cues. PAK may serve as a molecular coincidence detector that promotes actin polymerization at the tips of filopodia downstream of signals from both the small GTPase Cdc42 and Src family kinases. Precise subcellular localization of GTPase effectors whose activity is gated by Src phosphorylation may represent a versatile mechanism allowing GTPase activation to result in specific cell behaviors such as filopodial protrusion. These results are consistent with the function of PAK in the regulation of axon pathfinding (Newsome et al., 2000) and dendritic spine morphology (Boda et al., 2004). In addition to identifying Src family kinases and PAK as necessary components of the filopodial tip complex, our findings suggest that intracellular signaling cascades converge onto common downstream targets to produce specific growth cone responses to guidance cues.

\section{References}

Bentley D, Toroian-Raymond A (1986) Disoriented pathfinding by pioneer neurone growth cones deprived of filopodia by cytochalasin treatment. Nature 323:712-715.

Berg JS, Cammer M, Chinegwundoh JO, Dale BM, Cheney RE, Greenberg S (2002) Myosin- $\mathrm{X}$ is an unconventional myosin that undergoes intrafilopodial motility. Nat Cell Biol 4:469-477.

Boda B, Alberi S, Nikonenco I, Node-Langlois R, Jourdain P, Moosmayer M, Parisi-Jourdain L, Muller D (2004) The mental retardation protein PAK3 contributes to synapse formation and plasticity in hippocampus. J Neurosci 24:10816-10825.

Bokoch GM (2003) Biology of the p21-activated kinases. Annu Rev Biochem 72:743-781.

Buss F, Kendrick-Jones J, Lionne C, Knight AE, Cote GP, Paul Luzio J (1998) The localization of myosin VI at the golgi complex and leading edge of fibroblasts and its phosphorylation and recruitment into membrane ruffles of A431 cells after growth factor stimulation. J Cell Biol 143:1535-1545.

Chien CB, Rosenthal DE, Harris WA, Holt CE (1993) Navigational errors made by growth cones without filopodia in the embryonic Xenopus brain. Neuron 11:237-251.

Chong C, Tan L, Lim L, Manser E (2001) The mechanism of PAK activation. Autophosphorylation events in both regulatory and kinase domains control activity. J Biol Chem 276:17347-17353.

Davenport RW, Dou P, Rehder V, Kater SB (1993) A sensory role for neuronal growth cone filopodia. Nature 361:721-724.

de la Torre JR, Hopker VH, Ming GL, Poo MM, Tessier-Lavigne M, Hemmati-Brivanlou A, Holt CE (1997) Turning of retinal growth cones in a netrin-1 gradient mediated by the netrin receptor DCC. Neuron 19:1211-1224.

Gehler S, Gallo G, Veien E, Letourneau PC (2004) p75 neurotrophin receptor signaling regulates growth cone filopodial dynamics through modulating RhoA activity. J Neurosci 24:4363-4372.

Geiger B, Bershadsky A, Pankov R, Yamada KM (2001) Transmembrane crosstalk between the extracellular matrix-cytoskeleton crosstalk. Nat Rev Mol Cell Biol 2:793-805.

Gomez TM, Roche FK, Letourneau PC (1996) Chick sensory neuronal growth cones distinguish fibronectin from laminin by making substratum contacts that resemble focal contacts. J Neurobiol 29:18-34.

Gomez TM, Robles E, Poo M, Spitzer NC (2001) Filopodial calcium transients promote substrate-dependent growth cone turning. Science 291:1983-1987.

Gomez TM, Harrigan D, Henley J, Robles E (2003) Working with Xenopus spinal neurons in live cell culture. Methods Cell Biol 71:129-156.
Guirland C, Suzuki S, Kojima M, Lu B, Zheng JQ (2004) Lipid rafts mediate chemotropic guidance of nerve growth cones. Neuron 42:51-62.

Iwasaki Y, Gay B, Wada K, Koizumi S (1998) Association of the Src family tyrosine kinase Fyn with TrkB. J Neurochem 71:106-111.

Jones SB, Lu HY, Lu Q (2004) Abl tyrosine kinase promotes dendrogenesis by inducing actin cytoskeletal rearrangements in cooperation with Rho family small GTPases in hippocampal neurons. J Neurosci 24:8510-8521.

Kiosses WB, Daniels RH, Otey C, Bokoch GM, Schwartz MA (1999) A role for p21-activated kinase in endothelial cell migration. J Cell Biol 147:831-844

Kirchner J, Kam Z, Tzur G, Bershadsky AD, Geiger B (2003) Live-cell monitoring of tyrosine phosphorylation in focal adhesions following microtubule disruption. J Cell Sci 116:975-986.

Knoll B, Drescher U (2004) Src family kinases are involved in EphA receptor-mediated retinal axon guidance. J Neurosci 24:6248-6257.

Lanier LM, Gates MA, Witke W, Menzies AS, Wehman AM, Macklis JD, Kwiatkowski D, Soriano P, Gertler FB (1999) Mena is required for neurulation and commissure formation. Neuron 22:313-325.

Les Erickson F, Corsa AC, Dose AC, Burnside B (2003) Localization of a class III myosin to filopodia tips in transfected HeLa cells requires an actin-binding site in its tail domain. Mol Biol Cell 14:4173-4180.

Lewis AK, Bridgman PC (1992) Nerve growth cone lamellipodia contain two populations of actin filaments that differ in organization and polarity. J Cell Biol 119:1219-1243.

Li W, Lee J, Vikis HG, Lee SH, Liu G, Aurandt J, Shen TL, Fearon ER, Guan JL, Han M, Rao Y, Hong K, Guan KL (2004) Activation of FAK and Src are receptor-proximal events required for netrin signaling. Nat Neurosci 7:1213-1221.

Lin CH, Espreafico EM, Mooseker MS, Forscher P (1997) Myosin drives retrograde F-actin flow in neuronal growth cones. Neuron 16:769-782.

Liu G, Beggs H, Jurgensen C, Park HT, Tang H, Gorski J, Jones KR, Reichardt LF, Wu J, Rao Y (2004) Netrin requires focal adhesion kinase and Src family kinases for axon outgrowth and attraction. Nat Neurosci 7:1222-1232.

Ma YC, Huang J, Ali S, Lowry W, Huang XY (2000) Src tyrosine kinase is a novel direct effector of G proteins. Cell 102:635-646.

Mallavarapu A, Mitchison T (1999) Regulated actin cytoskeleton assembly at filopodium tips controls their extension and retraction. J Cell Biol 146:1097-1106.

Meriane M, Tcherkezian J, Webber CA, Danek EI, Triki I, McFarlane S, Bloch-Gallego E, Lamarche-Vane N (2004) Phosphorylation of DCC by Fyn mediates Netrin-1 signaling in growth cone guidance. J Cell Biol 167:687-698.

Millard TH, Sharp SJ, Machesky LM (2004) Signalling to actin assembly via the WASP (Wiskott-Aldrich syndrome protein)-family proteins and the Arp2/3 complex. Biochem J 380:1-17.

Ming G, Lohof AM, Zheng JQ (1997) Acute morphogenic and chemotropic effects of neurotrophins on cultured embryonic Xenopus spinal neurons. J Neurosci 17:7860-7871.

Newsome TP, Schmidt S, Dietzl G, Keleman K, Asling B, Debant A, Dickson BJ (2000) Trio combines with dock to regulate Pak activity during photoreceptor axon pathfinding in Drosophila. Cell 101:283-294.

Nobes CD, Hall A (1995) Rho, rac, and cdc42 GTPases regulate the assembly of multimolecular focal complexes associated with actin stress fibers, lamellipodia, and filopodia. Cell 81:53-62.

Ren XR, Ming GL, Xie Y, Hong Y, Sun DM, Zhao ZQ, Feng Z, Wang Q, Shim S, Chen ZF, Song HJ, Mei L, Xiong WC (2004) Focal adhesion kinase in netrin-1 signaling. Nat Neurosci 7:1204-1212.

Renkema GH, Pulkkinen K, Saksela K (2002) Cdc42/Racl-mediated activation primes PAK2 for superactivation by tyrosine phosphorylation. Mol Cell Biol 22:6719-6725.

Robles E, Huttenlocher A, Gomez TM (2003) Filopodial calcium transients regulate growth cone motility and guidance through local activation of calpain. Neuron 38:597-609.

Sasaki Y, Cheng C, Uchida Y, Nakajima O, Ohshima T, Yagi T, Taniguchi M, Nakayama T, Kishida R, Kudo Y, Ohno S, Nakamura F, Goshima Y (2002) Fyn and Cdk5 mediate semaphorin-3A signaling, which is involved in regulation of dendrite orientation in cerebral cortex. Neuron 35:907-920.

Sells MA, Pfaff A, Chernoff J (2000) Temporal and spatial distribution of activated Pak1 in fibroblasts. J Cell Biol 151:1449-1458.

Shekarabi M, Kennedy TE (2002) The netrin-1 receptor DCC promotes filo- 
podia formation and cell spreading by activating Cdc42 and Rac1. Mol Cell Neurosci 19:1-17.

Souopgui J, Solter M, Pieler T (2002) XPak3 promotes cell cycle withdrawal during primary neurogenesis in Xenopus laevis. EMBO J 21:6429-6439.

Svitkina TM, Bulanova EA, Chaga OY, Vignjevic DM, Kojima S, Vasiliev JM, Borisy GG (2003) Mechanism of filopodia initiation by reorganization of a dendritic network. J Cell Biol 160:409-421.

Thomas SM, Brugge JS (1997) Cellular functions regulated by Src family kinases. Annu Rev Cell Dev Biol 13:513-609.

Torres E, Rosen MK (2003) Contingent phosphorylation/dephosphorylation provides a mechanism of molecular memory in WASP. Mol Cell 11:1215-1227.

Vastrik I, Eickholt BJ, Walsh FS, Ridley A, Doherty P (1999) Sema3Ainduced growth-cone collapse is mediated by Rac1 amino acids 17-32. Curr Biol 9:991-998.

Wang FS, Wolenski JS, Cheney RE, Mooseker MS, Jay DG (1996) Function of myosin-V in filopodial extension of neuronal growth cones. Science 273:660-663.
Worley TL, Holt C (1996) Inhibition of protein tyrosine kinases impairs axon extension in the embryonic optic tract. J Neurosci 16:2294-2306.

Worley TL, Cornel E, Holt C (1997) Overexpression of c-src and n-src in the developing Xenopus retina differentially impairs axonogenesis. Mol Cell Neurosci 9:276-292.

Wu DY, Goldberg DJ (1993) Regulated tyrosine phosphorylation at the tips of growth cone filopodia. J Cell Biol 123:653-664.

Wu DY, Wang LC, Mason CA, Goldberg DJ (1996) Association of $\beta 1$ integrin with phosphotyrosine in growth cone filopodia. J Neurosci $16: 1470-1478$

Yuan XB, Jin M, Xu X, Song YQ, Wu CP, Poo MM, Duan S (2003) Signalling and crosstalk of Rho GTPases in mediating axon guidance. Nat Cell Biology 5:38-45.

Zheng JQ, Felder M, Connor JA, Poo MM (1994) Turning of nerve growth cones induced by neurotransmitters. Nature 368:140-144.

Zheng JQ, Wan JJ, Poo MM (1996) Essential role of filopodia in chemotropic turning of nerve growth cone induced by a glutamate gradient. J Neurosci 16:1140-1149. 